\title{
Both-edges representation of letter position in reading
}

\author{
Simon Fischer-Baum • Jonathan Charny • \\ Michael McCloskey
}

Published online: 21 September 2011

(C) Psychonomic Society, Inc. 2011

\begin{abstract}
The representations that underlie our ability to read must encode not only the identities of the letters in a word, but also their relative positions. In recent years, many new proposals have been advanced concerning the representation of letter position in reading, but the available data do not distinguish among the competing proposals; multiple theories, each positing a different letter position representation scheme, are compatible with the evidence. In this article, we report two experiments that used the illusory word paradigm (e.g., Davis \& Bowers, Journal of Experimental Psychology: Human Perception and Performance, 30: 923-941, 2004) to distinguish among alternative schemes for representing letter position in reading. The results support a scheme that uses both the beginning and the end of a word as anchoring points. This both-edges scheme has been implicated in letter position representation in spelling (Fischer-Baum, McCloskey, \& Rapp, Cognition, 115: 466-490, 2010), as well as in position representation in verbal working memory (Henson, Memory \& Cognition, 27: 915-927, 1999), suggesting that it may be a domaingeneral scheme for representing position in a sequence.
\end{abstract}

Electronic supplementary material The online version of this article (doi:10.3758/s13423-011-0160-3) contains supplementary material, which is available to authorized users.

\section{S. Fischer-Baum $(\square)$}

Beckman Institute, University of Illinois,

Urbana-Champaign,

405 North Mathews Street,

Urbana, IL 61801, USA

e-mail: fischerbaum@cogsci.jhu.edu

J. Charny $\cdot$ M. McCloskey

Department of Cognitive Science, Johns Hopkins University,

Baltimore, MD, USA
Keywords Letter position representation - Letter migration errors · Visual word identification · Reading $\cdot$ Orthographic input coding

The cognitive processes that recognize written words must consider not only the identities, but also the positions, of letters (e.g., CAT vs. ACT). In this article, we focus on the representation of letter position in reading. A letter's position might be encoded on the basis of the distance from the beginning (e.g., Coltheart, Rastle, Perry, Langdon, \& Ziegler, 2001) or the midpoint (Caramazza \& Hillis, 1990) of the word, the letter's role in an orthographic syllable (e.g., Perry, Ziegler, \& Zorzi, 2010), or the context of surrounding letters (e.g., Grainger, Granier, Farioli, Van Assche, \& van Heuven, 2006; Whitney, 2001), or according to some other representational scheme. For example, the A in CARPET could be represented as the second letter from the beginning of the word $(B+2)$, the nucleus of the first orthographic syllable $\left(\mathrm{N}_{\sigma 1}\right)$, or the letter appearing after $\mathrm{C}$ and before $\mathrm{R}$ (C_R).

Although recent studies have addressed letter position representation empirically (e.g., Davis \& Bowers, 2004, 2006; Gomez, Ratcliff, \& Perea, 2008; Grainger et al., 2006; Kinoshita \& Norris, 2009; Perea \& Lupker, 2003), the available evidence is compatible with a range of alternative theories. For example, the transposition priming effect-lexical decisions for words (e.g., SERVICE) are facilitated more by transposition primes (e.g., SEVRICE) than by substitution primes (e.g., SEDLICE) - can be accommodated by theories that posit various types of letter position representation (Norris, Kinoshita, \& van Casteren, 2010). Additional evidence is needed to discriminate among the schemes. 
In the domain of spelling, Fischer-Baum, McCloskey, and Rapp (2010) used letter perseveration errors to contrast position representation schemes. Two participants with acquired dysgraphia (CM and LSS) intruded letters from prior spelling responses into subsequent responses when spelling to dictation. For example, after writing FARM, CM intruded an A into his next response, writing BLUNT as BLANT; and after writing FRENCE (a misspelling of FRENCH), he intruded an $\mathrm{R}$ into EDGE, writing ERGE. More often than would be expected by chance, the perseverated letters occupied the same position in the prior and subsequent responses, when position was defined relative to the beginning or end of the word. For example, the intruded $\mathrm{R}$ in ERGE appears in the same beginningbased position $(\mathrm{B}+2)$ as the $\mathrm{R}$ in FRENCE, and the intruded $\mathrm{A}$ in BLUNT appears in the same end-based position (E-3) as in FARM. Perseverated letters that did not appear in exactly matching beginning- or end-based positions were more likely than would be expected by chance to occupy approximately matching positions (i.e., positions immediately adjacent to the exactly matching beginning- or end-based positions). Representational schemes other than beginning- and end-based schemes made no contributions to accounting for the positions occupied by the perseverated letters.

From these results, Fischer-Baum et al. (2010) argued for both-edges representations of letter position in spelling. According to this scheme, a letter's position is encoded relative to both the beginning and the end of the word, such that the $\mathrm{A}$ in CARPET has position representations $\mathrm{B}+2$ and E-5. To explain the systematic tendency toward approximate as well as exact position matches, FischerBaum et al. proposed that the position representations are graded, such that nearby positions (e.g., $\mathrm{B}+2$ and $\mathrm{B}+3$ ) have more similar representations than distant positions (e.g., $\mathrm{B}+2$ and $\mathrm{B}+5$ ).

The same logic can be used to probe the representation of letter position in reading. In the illusory word paradigm, participants view brief presentations of word pairs (e.g., STOP and SHIP) and are cued to report one of the words (e.g., SHIP). We refer to the cued and noncued words as the target and distractor, respectively. Erroneous responses often take the form of letter migrations, in which a letter from the distractor (e.g., the O in STOP) intrudes into the response (e.g., SHIP reported as SHOP). Distractor letters are more likely to migrate to the matching target position (as in the STOP-SHOP example) than to a nonmatching position. However, migrations to approximately matching positions (e.g., SHIP reported as SHOP, given the distractor SOAP) also occur more often than do migrations to more distant positions (see, e.g., Davis \& Bowers, 2004, 2006).

Although previous studies demonstrated that letters tend to migrate to matching positions, these results can be accommodated by theories that posit various letter position representation schemes. The migrating O in the error SHOP occupies the same position as the $\mathrm{O}$ in STOP according to beginning-based $(B+3)$, midpoint-based $(\bar{M}+1)$, and endbased (E-2) schemes, as well as by syllabic schemes $\left(\mathrm{N}_{\sigma 1}\right)$, and even some letter-context schemes, since both Os appear after an $\mathrm{S}$ and before a $\mathrm{P}$.

In the present study, we developed target-distractor pairs that allowed us to contrast positional schemes. Consider the migration error TUBE $\rightarrow$ TUNE. How often should this error occur with each of the following distractors: TANGLE, TRANCE, and THRONE? In the beginning-based distractor TANGLE, the $\mathrm{N}$ occupies the same beginning-based position as the $\mathrm{N}$ in TUNE $(\mathrm{B}+3)$. For the midpoint-based distractor TRANCE, the $\mathrm{N}$ matches with respect to midpoint-based position $(\mathrm{M}+1)$. For the end-based distractor THRONE, the $\mathrm{N}$ appears at the same end-based position (E-2).

Figure 1 depicts the predictions for beginning-, midpoint-, and end-based and both-edges positional schemes. If position is represented by a graded beginningbased scheme, the TUBE $\rightarrow$ TUNE error should occur most often with the beginning-based distractor TANGLE (because the $\mathrm{N}$ occupies position $\mathrm{B}+3$ in both TUNE and TANGLE), followed by with the midpoint-based distractor TRANCE $(\mathrm{B}+3$ vs. $\mathrm{B}+4)$, and finally with the end-based distractor THRONE $(\mathrm{B}+3$ vs. $\mathrm{B}+5)$. For a graded endbased scheme, the opposite pattern would be expected: TUBE $\rightarrow$ TUNE should occur most often with the endbased distractor THRONE (because the N's position is E-2 in both TUNE and THRONE), followed by with the midpoint-based distractor TRANCE (E-2 vs. E-3), and finally with the beginning-based distractor TANGLE (E-2 vs. E-4). For a graded midpoint-based scheme, the TUBE $\rightarrow$ TUNE error should be most likely with the midpoint-based distractor TRANCE (because the $\mathrm{N}$ occupies position $\mathrm{M}+1$ in both TUNE and TRANCE), and less likely with the beginning- and end-based distractors TANGLE $(\mathrm{M}+1$ vs. $\mathrm{M}-1)$ and THRONE $(\mathrm{M}+1$ vs. $\mathrm{M}+2)$.

Finally, if position is represented by a graded both-edges scheme, then TUBE $\rightarrow$ TUNE should occur frequently with the beginning-based distractor TANGLE (both $\mathrm{Ns}$ in position $\mathrm{B}+3$ ) and with the end-based distractor THRONE (both E-2), but less often with the midpoint-based distractor TRANCE (because the Ns in TUNE and TRANCE differ on both beginning- and end-based positions). However, the graded both-edges scheme nevertheless predicts above-baseline rates of TUBE $\rightarrow$ TUNE errors given TRANCE, because the Ns approximately match on both beginning-based $(\mathrm{B}+3$ vs. $\mathrm{B}+4)$ and end-based $(\mathrm{E}-2$ vs. E-3) positions.

Beginning-, midpoint-, and end-based distractors also provide a basis for contrasting other positional schemes. For a given migration error (e.g., TUBE $\rightarrow$ TUNE), beginning- 
Fig. 1 Predictions of four schemes for letter position representation concerning the likelihood of the TUBE $\rightarrow$ TUNE letter migration error given beginning-based (TANGLE), midpoint-based (TRANCE), and end-based (THRONE) distractors
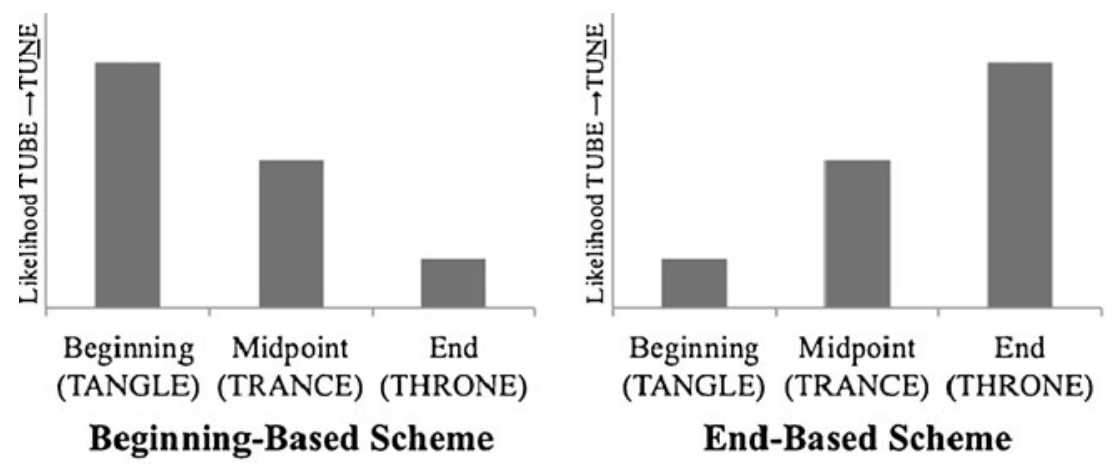

End-Based Scheme

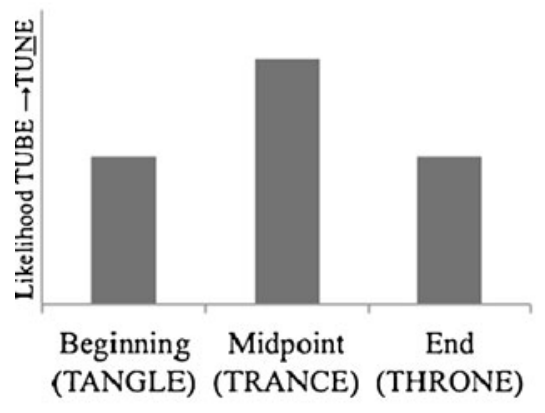

Midpoint-Based Scheme

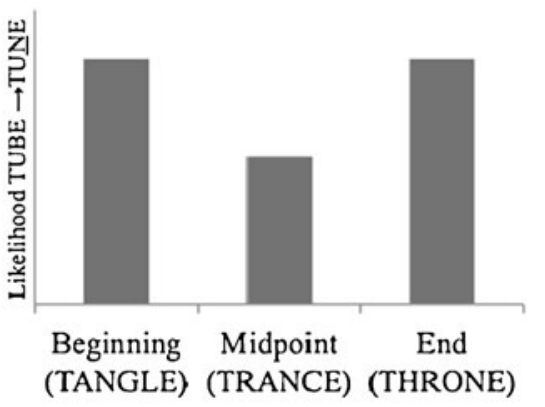

Both-Edges Scheme and end-based distractors can be categorized as either closeror farther-edge distractors. For the $\mathrm{N}$ in TUNE, the closer edge of the word is the end, and the farther edge is the beginning. For this migration error, the end-based distractor (THRONE) is the closer-edge distractor, and the beginningbased distractor (TANGLE) is the farther-edge distractor. In other words, the $\mathrm{N}$ in TUNE matches the $\mathrm{N}$ in THRONE with respect to closer-edge position, and matches the $\mathrm{N}$ in TANGLE with respect to farther-edge position. For other migration errors, such as SOME $\rightarrow$ SAME, the closer edge is the beginning and the farther edge is the end. For these errors, beginning-based distractors (e.g., SALUTE) are closer-edge distractors, and end-based distractors (e.g., SENATE) are farther-edge distractors.

Because each closer- and farther-edge distractor is either a beginning- or end-based distractor, the both-edges scheme predicts that closer- and farther-edge distractors should yield more frequent letter migration errors than would midpoint distractors. Other prominent theories of letter position representation predict fewer migration errors for farther-edge than for midpoint distractors, or no difference between the two distractor types (e.g., the spatial coding model, Davis, 2010; the overlap model, Gomez, Ratcliff, \& Perea, 2008; overlapping open bigrams, Grainger et al. 2006; and SERIOL, Whitney, 2008). These predictions rely on Davis and Bowers's (2006) assumption that the migration rate is a function of the overall similarity between the distractor and the error response. Each theory includes methods for calculating the similarity between two letter strings, and despite substantial differences in their assumptions about letter position representations, all of the theories imply that midpoint distractors (e.g., TRANCE) are at least

Table 1 The similarity between errors and either farther-edge or midpoint distractors for different theories of position representation in reading

\begin{tabular}{|c|c|c|}
\hline \multirow[t]{2}{*}{ Theory } & \multicolumn{2}{|l|}{ Similarity } \\
\hline & Error-Farther Edge & Error-Midpoint \\
\hline Spatial coding (Davis, 2010) & .69 & .71 \\
\hline Overlap model (Gomez et al. 2008) ${ }^{\mathrm{a}}$ & $.36 / .37$ & $.37 / .39$ \\
\hline Overlapping open bigrams (Grainger et al. 2006) & .13 & .18 \\
\hline SERIOL (Whitney, 2001, 2008) & .47 & .48 \\
\hline
\end{tabular}

See the supplemental materials for derivations of these values. ${ }^{\text {a }}$ Because the overlap model assumes a different degree of position uncertainty for Position 3 than for Position 2, different similarity scores were calculated for second-position (SAME vs. SENATE or SCARCE) and third-position (TUNE vs. TANGLE or TRANCE) migrations. However, in both cases the error was more similar to the midpoint than to the farther-edge distractor. 
as similar as, and in most cases more similar than, fartheredge distractors (e.g., TANGLE) to the corresponding migration error responses (e.g., TUNE). Table 1 presents the similarity score for each theory, and the Supplemental Materials describe how these values were calculated.

What is the locus of the letter migration errors? Davis and Bowers (2004) argued that migrations arise at a lexical level. The target and distractor sequences (e.g., STOP and SHIP) both spread activation to the same lexical representation (SHOP), which is occasionally produced. Treisman and Souther (1986) argued that migrations arise at a prelexical level. With brief presentation, a letter's identity and position within a sequence may be activated independently of the sequence in which the letter appeared. Understanding the level at which these errors arise allows for stronger inferences about the part of the system to which the results apply.

Below, we report two experiments examining letter migration rates as a function of distractor type. The experiments differ with respect to whether the critical migration errors result in pseudowords (Exp. 1) or words (Exp. 2). According to the lexical account, letter migrations should only lead to word errors, whereas the prelexical account can accommodate both word and nonword errors. We also varied response modality; participants either typed (Exp. 1) or spoke (Exp. 2) their responses. Similar results across modalities localize migration errors to the orthographic input system required for both tasks.

\section{Experiment 1}

\section{Method}

Participants A group of 30 students from Johns Hopkins University participated in the study for extra course credit. All were native English speakers with normal or correctedto-normal vision.

Materials The targets were 30 four-letter and 30 six-letter pseudowords. Each target was paired with four distractor pseudowords that began and ended with the same letter as the target. Four-letter targets were paired with six-letter distractors, and six-letter targets with four-letter distractors. The critical pairs for our analysis consisted of fourletter targets paired with six-letter distractors (e.g., $\mathrm{ABCD}-\mathrm{AxxxxD}$ ).

For each four-letter target, two substitution errors-one for the second and one for the third target letter-were predesignated as critical errors $(\mathrm{B} \rightarrow \mathrm{Y}$ and $\mathrm{C} \rightarrow \mathrm{Z}$ ). For each target stimulus, beginning-, midpoint-, and end-based distractors were constructed to elicit the critical substitutions via letter migration (see Table 2). In beginning-based distractors, the potential migrating letters appeared at positions $\mathrm{B}+2$ and $\mathrm{B}+3$ (AYZxxD), and so occupied the same beginning-based positions as the critical target letters $(\mathrm{B}$ and $\mathrm{C})$ in $\mathrm{ABCD}$. In midpoint-based distractors $(\mathrm{AxYZxD})$, the potential migrating letters appeared at positions $M-1$ and $M+1$ (third and fourth letters), and so matched the critical target letters with respect to midpointbased position; and in end-based distractors (AxxYZD), the potential migrating letters occupied positions $\mathrm{E}-3$ and $\mathrm{E}-2$ (fourth and fifth letters), matching the critical target letters on end-based position. Each target was also paired with a control distractor $(\mathrm{AxxxxD})$ that did not contain the potential migrating letters.

Procedure Participants were tested individually. A total of 6 practice trials and 240 experimental trials (60 targets $\times 4$ distractor types) were presented in a single session lasting approximately $20 \mathrm{~min}$. The experimental trials were divided into four 60-trial blocks, with each target appearing once per block. Each block also contained approximately equal numbers of trials with each of the four distractor types. Ordering of trials within blocks was random.

Each trial began with a fixation cross displayed at the center of a computer monitor for $1 \mathrm{~s}$. After a 500-ms blank interval, the target-distractor pair was presented for $200 \mathrm{~ms}$ in 24-point lowercase Courier font, with one stimulus to the left and the other to the right of the screen's center. The target appeared equally often in the left and right positions. Two character spaces separated the last letter of the left stimulus and the first letter of the right stimulus. Stimulus presentation was followed by a 200 -ms nonalphanumeric character mask. Upon mask offset, a line of the same length as the target appeared immediately beneath the location at which the target had appeared. Participants responded by typing the target word.

\section{Results and discussion}

Table 3 presents the rates of critical migration errors (e.g., $\mathrm{ABCD} \rightarrow \mathrm{A} \underline{\mathrm{YCD}}$ or $\mathrm{AB} \underline{\mathrm{ZD}}$ ) as a function of distractor type. The response to a target was scored as a critical migration error if the response had the correct number of letters (i.e., four) and included at least one of the critical letter substitutions (e.g., $\mathrm{B} \rightarrow \mathrm{Y}$ and/or $\mathrm{C} \rightarrow \mathrm{Z}$ ).

The effect of distractor type was significant in both participant and item ANOVAs, $F_{1}(3,119)=15.43, p<$ $.0001 ; F_{2}(3,119)=12.13, p<.0001 .{ }^{1}$ Post-hoc Tukey

\footnotetext{
${ }^{1}$ We arcsine transformed the data for all analyses and report untransformed means in the text and tables. For the beginning-, end-, and midpoint-based and control distractors, respectively, the arcsinetransformed means were $.239, .202, .149$, and .114 by items (Tukey $\mathrm{HSD}[.05]=.059)$ and $.235, .223, .159$, and .116 by participants (HSD $[.05]=.053)$.
} 
Table 2 Examples of stimuli from Experiments 1 and 2

\begin{tabular}{|c|c|c|c|c|c|c|}
\hline \multirow[t]{2}{*}{ Experiment } & \multirow[t]{2}{*}{ Target (ABCD) } & \multirow{2}{*}{$\begin{array}{l}\text { Migration Error } \\
(\mathrm{AYxD} \text { or } \mathrm{AxZD})\end{array}$} & \multicolumn{4}{|c|}{ Distractor Condition } \\
\hline & & & $\begin{array}{l}\text { Beginning } \\
\text { (AYZxxD) }\end{array}$ & $\begin{array}{l}\text { End } \\
\text { (AxxYZD) }\end{array}$ & $\begin{array}{l}\text { Midpoint } \\
\text { (AxYZxD) }\end{array}$ & $\begin{array}{l}\text { Control } \\
\text { (AxxxxD) }\end{array}$ \\
\hline Experiment 1 & STIN & SLIN or STEN & SLERON & SAFLEN & SOLERN & SPOBUN \\
\hline Experiment 2: Word Targets & FAST & FIST or FAC $\underline{T}$ & FICROT & FOPICT & FLICUT & FALMUD \\
\hline Experiment 2: Pseudoword Targets & TUSE & TUUNE & $\overline{T A N G L E}$ & TRANCE & THRONE & TRIFLE \\
\hline
\end{tabular}

analyses revealed that the migration error rate was significantly higher for beginning- and end-based distractors $(6.3 \%$ and $5.6 \%$, respectively) than for control distractors $(1.4 \%)$, $p$ s $<.01$. In contrast, midpoint-based distractors $(2.9 \%)$ did not differ from control. Furthermore, the migration rate was significantly lower for midpoint-based distractors than for beginning- or end-based distractors, although the difference between end and midpoint distractors only reached significance in the by-participants analysis $(p s<.01)$. No other differences were significant, including the difference between beginning- and end-based distractors.

These findings match the predictions of the both-edges position representation scheme, but not of the beginning-, midpoint-, or end-based schemes (see Fig. 1). The data do not provide clear evidence for a graded version of the both-edges scheme, because the difference between midpoint and control distractors predicted by the graded scheme was not statistically significant. However, caution should be exercised in interpreting this result, since migration rate was numerically higher for midpoint $(2.9 \%)$ than for control distractors $(1.4 \%)$.

A second analysis involved the farther-edge distractors $(\mathrm{AYZxxD}$ for $\mathrm{ABCD} \rightarrow \mathrm{ABZD}$ and $\mathrm{Axx} \underline{\mathrm{ZDD}}$ for $\mathrm{ABCD} \rightarrow$ $A Y C D)$. As predicted by the both-edges scheme, the rate of critical migration errors was higher for the farther-edge distractors $(5.2 \%)$ than for the midpoint distractors $(2.9 \%)$, $t(29)=4.67, p<.0001$, in the participants analysis and $t(29)=$ 2.86, $p<.01$, in the items analysis (both one-tailed tests). ${ }^{2}$ These results support the both-edges scheme and pose a problem for the spatial coding, overlap, overlapping open bigrams, and SERIOL models, all of which predict either no difference between the distractor types or higher migration rates for midpoint than for farther-edge distractors.

\section{Experiment 2}

This experiment explored the generality of the results from Experiment 1. Unlike in the previous experiment, the distractor and target stimuli included real words in addition

\footnotetext{
$\overline{2}$ Arcsine-transformed means by participants: farther edge $=.208$, midpoint $=.149$; by items: farther edge $=.220$, midpoint $=.159$.
}

to pseudowords, participants gave spoken instead of typed responses, and the critical migration errors were real words rather than pseudowords.

\section{Method}

The targets were 30 pseudowords and 30 words (half four letters and half six letters long). Each target was paired with four distractors, such that each target-distractor pair had one four- and one six-letter string and one familiar word and one pseudoword. Given the constraints imposed by the use of real words as targets, distractors, and critical migration errors, we were able to construct only 23 critical target-distractor pairs ( 15 word-pseudoword and 8 pseudoword-word pairs). All critical pairs consisted of four-letter targets and six-letter distractors.

For each critical word target (e.g., FAST), two substitution errors - one for the second and one for the third target letterwere predesignated as critical errors (e.g., FIST, FAC $\underline{\text { T }}$ ), and beginning-, midpoint-, and end-based distractors were constructed to elicit the critical substitutions via letter migration (see Table 2). For each critical pseudoword target (e.g., TUSE), single substitution errors for the second and third target letters were predesignated as critical errors (e.g., TUNE), and beginning-, midpoint-, and end-based distractors were constructed to elicit the critical error (see Table 2).

The participants were 72 Johns Hopkins University students. The procedures were the same as in Experiment 1 , except that responses were spoken rather than typed.

Results and discussion

Table 3 presents the rates of critical migration errors for each distractor type. No differences were observed in error rates between word and pseudoword targets $(F \mathrm{~s}<1)$. Subsequent analyses were collapsed across target lexicality. ANOVAs showed a significant effect of distractor type, $F_{1}(3,287)=$ 22.30, $p<.0001 ; F_{2}(3,91)=8.67, p<.0001 .^{3}$ Post-hoc

\footnotetext{
${ }^{3}$ The arcsine-transformed means for the beginning-, end-, and midpoint-based and control distractors were .193, .180, .146, and .090 by items $(\operatorname{HSD}[.05]=.058)$ and $.216, .207, .175$, and .125 by participants $(\mathrm{HSD}[.05]=.032)$.
} 
Table 3 Migration rates in Experiments 1 and 2 for each distractor condition (standard errors by participants)

\begin{tabular}{lll}
\hline Distractor Type & \multicolumn{2}{l}{ Percent Migration Rate (Standard Error) } \\
\cline { 2 - 3 } & Experiment 1 & Experiment 2 \\
\hline Beginning & $6.3(0.9)$ & $5.0(0.5)$ \\
End & $5.6(0.8)$ & $4.6(0.5)$ \\
Midpoint & $2.9(0.5)$ & $3.1(0.5)$ \\
Control & $1.4(0.3)$ & $0.8(0.2)$ \\
\hline
\end{tabular}

Tukey analyses revealed significantly higher migration rates for beginning- $(5.0 \%)$ and for end- $(4.6 \%)$ based than for control distractors $(0.8 \%), p \mathrm{~s}<.01$, by both items and participants. By participants, the migration error rate was significantly higher for the midpoint $(3.1 \%)$ than for the control $(0.8 \%)$ distractors, and lower than the rates for the beginning $(5.0 \%)$ and end $(4.6 \%, p \mathrm{~s}<.05)$ distractors.

These results support the both-edges position representation scheme over the beginning-, midpoint-, and endbased schemes (see Fig. 1). Additionally, the finding that migration errors were significantly more frequent for midpoint than for control distractors is consistent with a graded both-edges scheme.

A second analysis compared farther-edge and midpoint distractors. As predicted by the both-edges scheme, the migration rate was higher for farther-edge (2.1\%) than for midpoint (1.6\%) distractors. This difference was significant by participants, $t(71)=1.92, p<.05$, though not by items. ${ }^{4}$ These results replicate the findings from Experiment 1, supporting the both-edges scheme, and are problematic for the spatial coding, overlap, overlapping open bigrams, and SERIOL models.

\section{General discussion}

In the illusory word paradigm, brief presentations of targetdistractor pairs (e.g., STOP-SHIP) frequently result in letter migration errors, in which a letter from the distractor appears in the response to the target (e.g., SHOP). Although previous studies (e.g., Davis \& Bowers, 2004, 2006) demonstrated that distractor letters tend to migrate to matching target positions (as in migration of the $\mathrm{O}$ from STOP to SHOP), these results are consistent with a variety of representational schemes for encoding letter position. In the present study, we contrasted position representation schemes in two experiments using the illusory word paradigm. Results from both experiments supported a

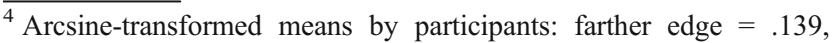
midpoint $=.123$.
}

both-edges scheme for letter position representation. This scheme encodes a letter's position relative to both the beginning and the end of the word, such that, for example, the $\mathrm{O}$ in STOP is assigned position representations $\mathrm{B}+3$ and E-2. Our results also suggested that the position representations are graded, such that nearby positions (e.g., $\mathrm{B}+2$ and $\mathrm{B}+3$ ) have more similar representations than distant positions (e.g., $\mathrm{B}+2$ and $\mathrm{B}+5$ ).

The results of our experiments are problematic for alternative schemes of representing letter position in reading, including beginning-based (e.g., Coltheart et al. 2001), midpoint-based (e.g., Caramazza \& Hillis, 1990), context-dependent (e.g., Grainger et al. 2006; Whitney, 2001), and other proposed schemes (overlap model, Gomez et al., 2008; spatial coding model, Davis, 2010). The experiments did not directly address syllabic schemes (e.g., Perry et al. 2010); we leave the comparison between bothedges and syllabic schemes for future research.

Many of these alternative positional schemes are used in theories that account for vast amounts of empirical data. For example, Davis (2010) lists more than 60 benchmark results, and with a remarkable fit, his spatial coding model explains them all. Given the impressive successes of these theories, what should be made of their failure to explain the present results?

One possibility is that the present findings are outside the scope of theories that posit positional schemes other than the both-edges scheme. The predictions these theories generate are typically based on the degree to which a lexeme (e.g., STOP) is activated by a stimulus (e.g., SHOP), and therefore reflect a lexical-level orthographic representation. However, the present results suggest a prelexical locus of migration errors; similar results were obtained whether or not a migration error resulted in a word. These errors may reflect the nature of position information at a level of representation that the theories discussed above do not address. However, recent research has suggested that other benchmark results also arise from prelexical representations (Kinoshita \& Norris, 2009); ignoring prelexical orthographic representations may be a real limitation of these theories.

Alternatively, the successes of the theories may have little to do with their specific choice of representation scheme. Theories that use different position representation schemes accommodate many of the same benchmark results. Some results appear unrelated to issues of position representation (e.g., frequency effects), and other findings that speak to position representation can nevertheless be accommodated by theories that posit various schemes. For example, almost any graded representation scheme can account for the transposition priming effect (e.g., SERVICE is primed more by SEVRICE than by SEDLICE), because adjacent letters have similar position representations in graded schemes. 
These considerations suggest that in addition to explaining the results of the present study, theories adopting graded both-edges positional schemes could conceivably be as successful as extant theories in accounting for Davis's (2010) benchmark results. Indeed, some of these results seem to fit well with a both-edges scheme. For example, Grainger et al. (2006) found that lexical decision to a target word is facilitated by primes consisting only of the initial (beginning-based position matches) or final (end-based position matches) letters. We are currently exploring whether a theory that uses a graded both-edges scheme can account for the available evidence.

Finally, the present results, in conjunction with the Fischer-Baum et al. (2010) findings from spelling, suggest that reading and spelling rely on the same graded bothedges scheme. This result is consistent with theories holding that reading and spelling rely on shared representations of letter identity and position (e.g., Caramazza \& Hillis, 1990). Evidence for graded both-edges representations has also been reported in other cognitive domains. Henson (1999) argued for a both-edges representation of item position in immediate serial recall. The both-edges scheme may be a domain-general scheme for representing the positions of elements in a sequence.

Author Note This research was supported by fellowships from the William Orr Dingwall Foundation and the Arnold and Mabel Beckman Foundation granted to the first author. We thank Brenda Rapp and Carol Whitney for useful discussions concerning this work. Portions of this work were presented at the 50th Annual Meeting of the Psychonomic Society. Supplemental materials may be downloaded along with this article from www.springerlink.com.

\section{References}

Caramazza, A., \& Hillis, A. E. (1990). Levels of representation, coordinate frames, and unilateral neglect. Cognitive Neuropsychology, 7, 391-445.

Coltheart, M., Rastle, K., Perry, C., Langdon, R., \& Ziegler, J. (2001). DRC: A dual route cascaded model of visual word recognition and reading aloud. Psychological Review, 108, 204-256. doi:10.1037/0033-295X.108.1.204
Davis, C. J. (2010). The spatial coding model of visual word identification. Psychological Review, 117, 713-758.

Davis, C. J., \& Bowers, J. S. (2004). What do letter migration errors reveal about letter position coding in visual word recognition? Journal of Experimental Psychology: Human Perception and Performance, 30, 923-941. doi:10.1037/0096-1523.30.5.923

Davis, C. J., \& Bowers, J. S. (2006). Contrasting five different theories of letter position coding: Evidence from orthographic similarity effects. Journal of Experimental Psychology: Human Perception and Performance, 32, 535-557. doi:10.1037/00961523.32.3.535

Fischer-Baum, S., McCloskey, M., \& Rapp, B. (2010). The representation of letter position in spelling: Evidence from acquired dysgraphia. Cognition, 115, 466-490.

Gomez, P., Ratcliff, R., \& Perea, M. (2008). The overlap model: A model of letter position coding. Psychological Review, 115, $577-$ 600. doi:10.1037/a0012667

Grainger, J., Granier, J. P., Farioli, F., Van Assche, E., \& van Heuven, W. (2006). Letter position information and printed word perception: The relative-position priming constraint. Journal of Experimental Psychology: Human Perception and Performance, $32,865-884$.

Henson, R. N. A. (1999). Positional information in short-term memory: relative or absolute? Memory \& Cognition, 27, 915927.

Kinoshita, S., \& Norris, D. (2009). Transposed-letter priming of prelexical orthographic representations. Journal of Experimental Psychology: Learning, Memory, and Cognition, 35, 1-18. doi:10.1037/a0014277

Norris, D., Kinoshita, S., \& van Casteren, M. (2010). A stimulus sampling theory of letter identity and order. Journal of Memory and Language, 62, 254-271. doi:10.1016/j.jml.2009.11.002

Perea, M., \& Lupker, S. J. (2003). Transposed-letter confusability effects in masked form priming. In S. Kinoshita \& S. J. Lupker (Eds.), Masked priming: The state of the art (pp. 97-120). Hove, U.K.: Psychology Press.

Perry, C., Ziegler, J. C., \& Zorzi, M. (2010). Beyond single syllables: Large-scale modeling of reading aloud with the connectionist dual process (CDP++) model. Cognitive Psychology, 61, 106-151. doi:10.1016/j.cogpsych.2010.04.001

Treisman, A., \& Souther, J. (1986). Illusory words: The roles of attention and of top-down constraints in conjoining letters to form words. Journal of Experimental Psychology: Human Perception and Performance, 12, 3-17. doi:10.1037/0096-1523.12.1.3

Whitney, C. (2001). How the brain encodes the order of letters in a printed word: The SERIOL model and selective literature review. Psychonomic Bulletin \& Review, 8, 221-243. doi:10.3758/ BF03196158

Whitney, C. (2008). Comparison of the SERIOL and SOLAR theories of letter-position encoding. Brain and Language, 107, 170-178. doi:10.1016/j.bandl.2007.08.002 